\title{
Cerebral blood flow during vasovagal syncope induced by active standing or head up tilt
}

\author{
R Y T Sung, Z D Du, C W Yu, M C Yam, T F Fok
}

\begin{abstract}
Background-Vasovagal syncope is usually associated with a sudden drop of blood pressure and/or heart rate. However, occasionally the symptoms of syncope induced by orthostatic stress testing are not associated with obvious haemodynamic changes. The mechanisms of syncope in these patients are not clear. Aim-To evaluate changes in cerebral blood flow velocities during orthostatic stress testing in children and adolescents with vasovagal syncope.

Methods-Electrocardiogram, instantaneous arterial blood pressure, and right middle cerebral artery blood flow velocity were recorded at rest, during active standing, and $80^{\circ}$ head up tilt. 32 children and adolescents aged between 7 and 18 years with a history of repeated vasovagal syncope and 23 healthy control subjects were studied.

Results-Presyncope occurred in 10 patients during standing, and 13 patients during head up tilt. None of the controls had symptoms during the test. The transcranial Doppler study showed that the symptoms were associated with significant decreases of diastolic cerebral blood flow velocity and an increase of pulsatility. There was no significant change of the systolic cerebral blood flow velocity. The changes of cerebral blood flow velocities occurred in all episodes of presyncope, including those not associated with severe drop of blood pressure or heart rate. Conclusions-Diastolic cerebral blood flow velocity decreased significantly during episodes of presyncope induced by orthostatic stress. Impairment of autoregulation of cerebral blood flow might play an important role in the pathophysiology of syncope.

(Arch Dis Child 2000;82:154-158)
\end{abstract}

Department of Paediatrics, Prince of Wales Hospital, Shatin, Hong Kong, The People's Republic of China

R Y T Sung

Z D Du

C W Yu

M C Yam

T F Fok

Correspondence to: Dr Sung email: yntzsung@ cuhk.edu.hk

Accepted 23 September 1999
Keywords: autoregulation; cerebral blood flow; syncope; transcranial Doppler

The most common form of syncope encountered in children and adolescents is vasovagal or neurally mediated syncope. ${ }^{12}$ The head up tilt and the active standing tests are often used to support the diagnosis, based on the reproduction of symptoms of syncope or presyncope concomitant with systemic hypotension and/or bradycardia..$^{3-5}$ However, we have occasionally encountered children who developed symptoms of syncope or presyncope not associated with a significant decrease of blood pressure or heart rate during standing or head up tilt testing. We have had difficulty in the interpretation of these results. Recently, a few case series reports have enlightened us by showing the same phenomenon in adult patients and demonstrating reduced cerebral blood flow by transcranial Doppler study during the head up tilt testing. ${ }^{6}{ }^{7}$ It was hypothesised that impairment of cerebrovascular autoregulation might be the cause of syncope in these patients. ${ }^{89}$

To our knowledge, there has been no report on transcranial Doppler study of cerebral blood flow velocity during orthostatic stress in children and adolescents. To understand the mechanism of vasovagal syncope in children better, we adopted the transcranial Doppler method to compare the changes of cerebral blood flow velocity during head up tilt and active standing in children and adolescents who had recurrent vasovagal syncope with that in normal controls.

\section{Methods}

SUBJECTS

From March to November 1998, consecutive patients aged between 7 and 18 years old with a clinical diagnosis of recurrent vasovagal syncope in a university hospital in Hong Kong were recruited to our study. The diagnosis of vasovagal syncope was made by obtaining a typical history of fainting from the upright position with marked pallor and sweating thereafter, and by exclusion of other causes of syncope. ${ }^{110}$ Congenital or acquired heart disease and cardiac arrhythmia were excluded by a complete physical examination, 12 lead electrocardiography, and echocardiography. We did not include any children who ever had seizures or abnormal electroencephalography (EEG). Other relevant laboratory investigations, including Holter monitoring, computed tomography, and magnetic resonance imaging, were carried out when necessary. None of the study children took medication of any kind before or during the examination.

Twenty three healthy volunteers within the same age range served as the control group. They were students attending local primary and secondary schools, and had no history of syncope or presyncope. Informed consent was obtained from both the child and a parent. Our study was approved by the clinical research ethics committee of the Chinese University of Hong Kong.

PROTOCOL

The tests were performed two hours after a light breakfast or lunch without coffee or tea in a room with a constant ambient temperature of 
$23^{\circ} \mathrm{C}$. Before the test, an explanation of the procedure was given. The stand up testing was performed after a rest at supine position for 10 minutes, followed by motionless standing for 15 minutes. If near syncope (lightheadedness, nausea, blurred vision, or sweating) or syncope occurred, the child was put back to supine position immediately.

After the stand up test and another five minutes' supine rest, the $80^{\circ}$ tilt up test with foot support was performed for 30 minutes or until the subject experienced signs of presyncope or syncope.

\section{MEASUREMENTS AND ANALYSIS}

Beat to beat blood pressures were continuously recorded from the third finger of the right hand with a volume clamp photoplethysmography (Finapres; Ohmeda, Englewood, Colorado, USA). ${ }^{11}$ The right arm was maintained at the level of the heart with a stand adjusted at heart level. Blood flow of the middle cerebral artery (MCA) was recorded by using a neuroflow portable transcranial Doppler system (Neuroguard, Freemont, California, USA). MCA was insonated through the temporal window at depths ranging between 25 and $45 \mathrm{~mm}$ with a $2 \mathrm{MHz}$ continuous Doppler probe. Electrocardiography was also monitored. The analogue data were input to a PC equipped with an analogue/digital acquisition system (MacLab; AD Instrument, Castle Hill, Australia) for later analysis.

Systolic (SBP), diastolic (DBP), and mean (MBP) blood pressures, heart rate (HR), MCA systolic (MCAs), diastolic (MCAd), and mean (MCAm) flow velocities, and MCA pulsatility index (PI; PI = MCAs - MCAd/MCAm) were derived off line and averaged for five minutes at baseline supine and five minutes after standing or head up tilt. All parameters were continuously recorded at syncope or presyncope.

STATISTICS

Data were presented as mean (SD). Values of heart rate, blood pressure, and cerebral blood flow velocity were compared using the paired $t$ test to determine whether there was a significant change between supine and standing or tilting. The changes of values at standing and head up tilt expressed as differences from base- line supine measurements were compared between the patients with syncope and normal controls. A $p$ value of $<0.05$ was used to determine significance.

\section{Results}

There were no significant differences between patients with syncope and normal controls in mean (SD) age (12.2 (2.8) v 12.0 (2.6) years), height $(151.3(14.6) v 147.5(16.2) \mathrm{cm})$, and weight (41.3 (12.3) $v 38.6(12.7) \mathrm{kg})$. There were more girls in the patient group (boys:girls, $9: 23$ ) than the control group (boys:girls, 12:11). No significant difference in sex, age, height, or weight was found between patients who developed symptoms during the testing and those who did not (all $\mathrm{p}>0.05$ ).

PRESENTATION OF SYMPTOMS

During the standing test, 10 of the 32 patients developed symptoms and signs of presyncope or near fainting such as nausea, pallor, sweating, and intolerance to standing. The symptoms occurred four to 15 minutes (mean, $8 ; \mathrm{SD}, 4)$ after standing. During the head up tilt test, 13 patients developed near fainting symptoms. The symptoms occurred two to $28 \mathrm{~min}-$ utes (mean, 11; SD, 9) after tilt. None of the controls had any symptoms.

HAEMODYNAMIC CHANGES DURING STANDING AND HEAD UP TILT

In both patients and normal controls the cardiovascular responses to active standing and $80^{\circ}$ head up tilt consisted of an increase in heart rate, DBP, and MBP. SBP was only significantly increased during active standing (table 1). There were no differences between the two groups in the changes of SBP, DBP, and heart rate during standing or tilting compared with supine baseline values (all $\mathrm{p}>0.05)$.

At presyncope, the SBP, DBP, MBP, and heart rate decreased significantly compared with corresponding baseline standing or head up tilt values (table 2). In the 10 patients who developed symptoms during standing, five showed a dramatic decrease of blood pressure, with SBP dropping to $50-62 \mathrm{mmHg}$ and DBP to $23-50 \mathrm{mmHg}$, and a simultaneous severe decrease in heart rate ( $>30$ beats/minute). Three had a severe drop of blood pressure

Table 1 Changes of blood pressure, heart rate, and cerebral blood flow velocities from supine and five minutes after standing or tilting in 32 patients with syncope and 23 normal controls

\begin{tabular}{|c|c|c|c|c|c|c|c|c|c|c|c|c|c|c|}
\hline \multirow[b]{4}{*}{$\mathrm{SBP}(\mathrm{mmHg})$} & & & & & \multicolumn{10}{|c|}{ Changes from supine value } \\
\hline & \multicolumn{4}{|c|}{ Supine } & \multicolumn{4}{|c|}{ Standing for five minutes } & \multirow[b]{2}{*}{$p$ Value } & \multicolumn{4}{|c|}{ Tilting for five minutes } & \multirow[b]{2}{*}{$p$ Value } \\
\hline & \multicolumn{2}{|c|}{ Patient } & \multicolumn{2}{|c|}{ Control } & \multicolumn{2}{|c|}{ Patient } & \multicolumn{2}{|c|}{ Control } & & \multicolumn{2}{|c|}{ Patient } & \multicolumn{2}{|c|}{ Control } & \\
\hline & 111 & (13) & 112 & (15) & 7 & (10) & 12 & (9) & NS & 1 & $(14)$ & 3 & (12) & NS \\
\hline $\mathrm{DBP}(\mathrm{mmHg})$ & 64 & (10) & 66 & (10) & 11 & (6) & 16 & (7) & NS & 7 & (10) & 7 & (10) & NS \\
\hline $\mathrm{MBP}(\mathrm{mmHg})$ & 80 & (11) & 80 & (8) & 11 & (7) & 12 & (6) & NS & 5 & (9) & 8 & (9) & NS \\
\hline Heart rate (bpm) & 79 & (10) & 77 & (13) & 14 & (12) & 12 & $(11)$ & NS & 14 & (11) & 9 & (10) & NS \\
\hline $\mathrm{MCAs}(\mathrm{cm} / \mathrm{s})$ & 64 & (22) & 66 & (21) & -1 & (9) & -5 & (9) & NS & -6 & (8) & 1 & (6) & NS \\
\hline $\operatorname{MCAd}(\mathrm{cm} / \mathrm{s})$ & 30 & (11) & 33 & (20) & -2 & (5) & -4 & (5) & NS & -4 & (5) & -1 & (3) & NS \\
\hline $\operatorname{MCAm}(\mathrm{cm} / \mathrm{s})$ & 42 & (15) & 44 & (21) & -3 & (6) & -4 & (6) & NS & -5 & (6) & 0 & (5) & NS \\
\hline PI (unit) & 0.7 & $8(0.12)$ & 0.7 & $4(0.12)$ & 0. & $7(0.12)$ & 0.0 & $7(0.12)$ & NS & 0. & $9(0.12)$ & 0.0 & $4(0.11)$ & NS \\
\hline
\end{tabular}

Data presented as mean (SD).

bpm, beats/minute; DBP, diastolic blood pressure; MBP, mean blood pressure; MCAd, middle cerebral artery diastolic blood flow velocity; MCAm, middle cerebral artery mean blood flow velocity; MCAs, middle cerebral artery systolic blood flow velocity; NS, not significant; PI, pulsatility index; SBP, systolic blood pressure. 
The blood pressure, heart rate, and cerebral flow velocities in patients at presyncope during standing and head up

\begin{tabular}{|c|c|c|c|c|c|}
\hline \multirow[b]{3}{*}{$\mathrm{SBP}(\mathrm{mmHg})$} & \multicolumn{2}{|l|}{ Standing $(n=10)$} & \multicolumn{3}{|c|}{ Head up tilt $(n=11)$} \\
\hline & Before presyncope & At presyncope & Before presyncope & \multicolumn{2}{|c|}{ At presyncope } \\
\hline & 123 & $(22)^{\star \star \star}$ & (21) & 85 & $(19)^{\star \star \star}$ \\
\hline $\mathrm{DBP}(\mathrm{mmHg})$ & (12) & $(21)^{\star}$ & (17) & 55 & $(15)^{\star}$ \\
\hline $\mathrm{MBP}(\mathrm{mmHg})$ & (13) & $(23)^{\star \star}$ & (17) & 67 & $(17)^{\star}$ \\
\hline Heart rate $(\mathrm{bpm})$ & (15) & $(26)^{\star}$ & (15) & 86 & $(22)^{\star}$ \\
\hline MCAs $(\mathrm{cm} / \mathrm{s})$ & (21) & (17) & $(24)$ & 58 & $(21)$ \\
\hline $\operatorname{MCAd}(\mathrm{cm} / \mathrm{s})$ & (10) & $(7)^{\star \star \star}$ & (14) & 11 & $(7)^{\star \star \star}$ \\
\hline $\operatorname{MCAm}(\mathrm{cm} / \mathrm{s})$ & $37 \quad(13)$ & $(8)^{\star \star}$ & (17) & 26 & $(10)^{\star \star}$ \\
\hline PI (unit) & $0.88(0.16)$ & $1.88(0.79)^{\star \star \star}$ & $0.93(0.11)$ & & $(0.66)^{\star \star \star}$ \\
\hline
\end{tabular}

Data presented as mean (SD).

Compared with before presyncope: ${ }^{\star} \mathrm{p}<0.05 ;{ }^{\star \star} \mathrm{p}<0.01 ;{ }^{\star \star \star} \mathrm{p}<0.001$

bpm, beats/minute; DBP, diastolic blood pressure; MBP, mean blood pressure; MCAd, middle cerebral artery diastolic blood flow velocity; MCAm, middle cerebral artery mean blood flow velocity; MCAs, middle cerebral artery systolic blood flow velocity; PI, pulsatility index; SBP, systolic blood pressure.

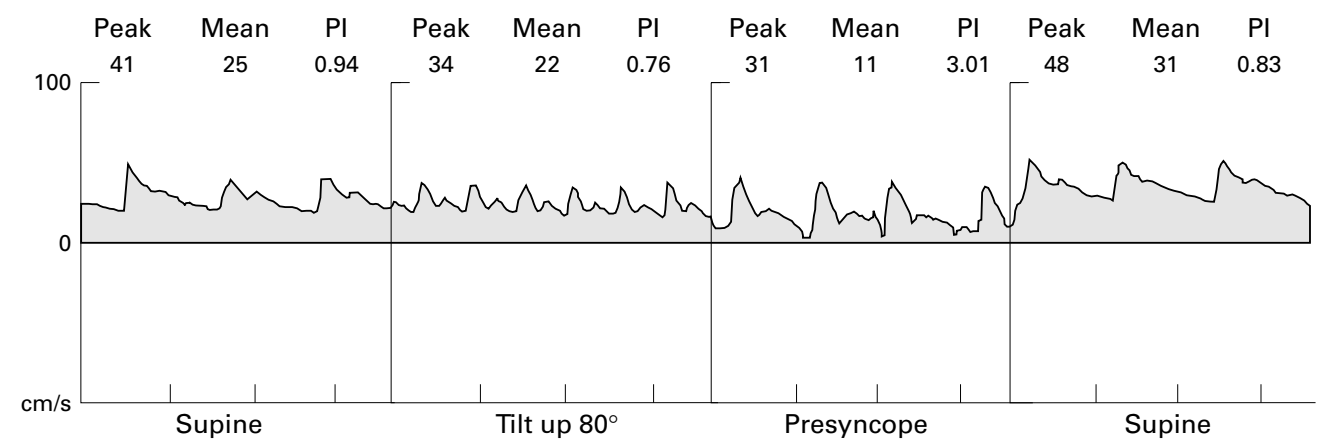

Figure 1 Transcranial Doppler waveform of right middle cerebral artery at a depth of $35 \mathrm{~cm}$ recorded during standing before syncope and at presyncope of a child with vasovagal syncope. At presyncope, there was a noticeable decrease of diastolic cerebral velocity and an increase of pulsatility index (PI).

alone. None had isolated bradycardia with a normal blood pressure. Two showed no obvious drop of blood pressure $(<20 \mathrm{mmHg})$ or heart rate. All 10 patients also developed symptoms of presyncope during $80^{\circ}$ tilt. In the 13 patients who developed presyncope during head up tilt, blood pressure and heart rate decreased simultaneously in four. Six patients had a significant decrease in blood pressure only. One had a severe drop of heart rate only, and three had no obvious changes of either blood pressure or heart rate.

CEREBRAL FLOW CHANGES DURING STANDING AND HEAD UP TILT

Transcranial Doppler recordings were successfully monitored in all study children except in two patients who were not recorded during $80^{\circ}$ tilt because of technical problems. No significant changes of MCAs, MCAd, MCAm, and PI were observed during standing or head up tilt compared with supine baseline values (table 1 ). The changes of the cerebral flow velocities did not differ between patients and normal controls either.

MCAd and MCAm decreased significantly, and PI increased significantly at the time of presyncope in all 10 patients who developed symptoms during standing, and all 11 patients during head up tilt in whom the cerebral flow at the time of presyncope was recorded. There was no significant change in MCAs (table 2). Compared with the values before presyncope, MCAd decreased by half whereas PI doubled at the time of presyncope. There were five episodes of presyncope that occurred in three patients (both at standing and $80^{\circ}$ tilt in two patients, only at tilt in one patient) without a significant decrease of blood pressure and heart rate; decreased MCAd and increased PI were recorded in four episodes. The MCA flow velocity at presyncope was not recorded in one episode because of a technical problem. In the only patient with a cardioinhibitory response during head up tilt, MCAd decreased from $20 \mathrm{~cm} / \mathrm{sec}$ to $12 \mathrm{~cm} / \mathrm{sec}$, and PI increased from 1.0 to 1.89 units. The most striking change identified at the time of presyncope in all cases was the decrease of MCAd. Figure 1 shows the Doppler cerebral blood flow before and at the time of presyncope during standing in one of the patients.

\section{Discussion}

Although vasovagal syncope is a common entity in paediatric clinical practice, its pathophysiology is still not completely understood..$^{12-14}$ Most investigators have emphasised the importance of an imbalance between sympathetic and parasympathetic systems, and the influence of biochemical mediators such as noradrenaline, angiotensin II, arginine-vasopressin, and nitric oxide. Transient hypotension and bradycardia are believed to result from an abnormal cardiovascular physiology, and to be the trigger of syncope. The haemodynamic response has been divided into three categories: vasodepression, cardiac inhibition, and combined. ${ }^{13}$ However, it has been reported in the adult population that some patients may present with presyncope or syncope in the absence of hypotension and bradycardia during orthostatic testing. ${ }^{67}$ Our study showed that this can also happen in children. 
Transcranial Doppler is a relatively new technique for assessment of the cerebral circulation. ${ }^{15} 16$ It can reliably record the cerebral blood flow velocities in various cerebral arteries, and permits non-invasive prolonged monitoring of rapidly changing cerebral blood flow. By measuring blood flow velocities in the MCA with transcranial Doppler, we found that MCAd and MCAm decreased, PI increased, and MCAs did not change at the time of presyncope during standing and head up tilt. This agrees with previous studies in adults. ${ }^{6-9}$ PI is an indicator of vascular resistance. ${ }^{15}{ }^{17} \mathrm{PI}$ increases when there is cerebral arteriolar vasoconstriction. Together with the ratio of systemic to diastolic velocity, it has a reported specificity of $98-100 \%$ in the diagnosis of vasospasm. ${ }^{15}{ }^{18}$ Therefore, our results could indicate that cerebrovascular resistance increased at the time of presyncope.

Cerebrovascular autoregulation occurs at the arteriolar level when arterial pressure changes. Cerebral arterioles dilate when pressure decreases and constrict when it increases. ${ }^{19}$ However, it was shown in our study that at syncope or presyncope, cerebral arterioles constrict concomitant with a decrease of arterial pressure in most of the cases. Grubb and colleagues have described this phenomenon and called it paradoxical vasoconstriction. They suggested that paradoxical vasoconstriction might play an important role in the development of vasovagal syncope. ${ }^{8}$

Similar to the findings of other groups, we found that in some patients a derangement in normal cerebrovascular autoregulation can result in cerebral arteriolar constriction in the absence of systemic hypotension or bradycardia and cause syncope. ${ }^{6720}$ We also noticed that MCAd and MCAm decreased and PI increased in four episodes of presyncope in the absence of hypotension or bradycardia. In our study, we stopped the test at the onset of presyncope and replaced the patient in the supine position because of ethical considerations. Therefore, it is possible that the drop of blood pressure and heart rate might have lagged behind the change of cerebral vascular flow velocity, but that we missed this. Nevertheless, it is very likely that disordered cerebral autoregulation, as an independent factor, might occur at the time of vasovagal syncope. Grossi and colleagues reported slow high amplitude, bilateral and synchronous $\Delta$ waves in patients who developed syncopal symptoms that were followed by convulsions during head up tilt testing. ${ }^{21}$ Grubb et al also reported that diffuse slow wave activity was detected on EEG in a patient who developed syncope during head up tilt testing. $\mathrm{He}$ demonstrated that the patient did not have systemic hypotension during the syncope, and that the EEG changes of cerebral hypoxia occurred concomitantly with a decrease of MCAd and an increase of PI. ${ }^{6}$ Therefore, we propose that in some patients abnormal baroreceptor responses triggered during orthostatic stress might result in a derangement of cerebral blood flow autoregulation, which might play an important role in the pathophysiology of vasovagal syncope.

During standing and head up tilt, the changes of blood pressure, heart rate, and cerebral blood flow velocities were similar in normal children and children with vasovagal syncope. This indicates that the autoregulation of the cerebral haemodynamics in response to orthostatic stress seems to be preserved except at the time of presyncope or syncope.

Cerebral circulation is autoregulated in a complicated way. It is possible that hyperventilation might lower the arterial carbon dioxide level and cause cerebral arteriolar constriction and syncope. We did not record the respiratory rate or expiratory carbon dioxide concentration in our study; however, obvious hyperventilation or hypoventilation was not noticed in any of the children during the standing and head up tilt tests.

In conclusion, our results suggest that cerebral autoregulation might be impaired in children with vasovagal syncope. The derangement occurs not only in children with systemic hypotension and bradycardia, but also in those without significant decreases of blood pressure and heart rate at the time of syncope or presyncope. The typical changes in cerebral blood flow velocity during vasovagal syncope are probably caused by the constriction of cerebral arteries. In addition to vasodepression and cardiac inhibition, cerebral vasoconstriction might be another mechanism in the pathogenesis of vasovagal syncope in children and adolescents.

1 Ruckmann RN. Cardiac causes of syncope. Pediatr Rev 1987;9:101-8.

2 Driscoll DJ, Jacobsen SJ, Porter CJ, et al. Syncope in children and adolescents. F Am Coll Cardiol 1997;29:103945 .

3 Benditt DG, Ferguson DW, Grubb BP, et al. Tilt table testing for assessing syncope (ACC expert consensus document). $\mathcal{F}$ Am Coll Cardiol 1996;28:263-75.

4 Kapoor WE, Smith MA, Miller NL. Upright tilt testing in evaluating syncope: a comprehensive literature review. $\mathrm{Am}$ Med 1994;97:78-88.

5 Tanaka H, Thulesius O, Yamaguchi O, Mino M. Circulatory responses in children with unexplained syncope evaluated by continuous non-invasive finger blood pressure monitorby continuous non-invasive finger b
ing. Acta Paediatr 1994;83:754-61.

6 ing. Acta Paediatr 1994;83:754-61. loss of consciousness associated with cerebral vasoconstriction in the absence of systemic hypotension. Pacing Clin Electrophysiol 1998;21:652-8.

7 Fredman CS, Biermann KM, Patel V, Uppstrom EL, Auer AI. Transcranial Doppler ultrasonography during headupright tilt-table testing. Ann Intern Med 1995;23:848-9.

8 Grubb BP, Gerard G, Roush K, et al. Cerebral vasoconstriction during head-upright tilt-induced vasovagal syncope: a paradoxic and unexpected response. Circulation 1991;84: 1157-64.

9 Stephenson JBP. Specific syncopes and anoxic seizure types. In: Stephenson JBP, ed. Fits and faints. London: Mackeith Press, 1990:67-70.

10 Bondar RL, Kassam MS, Stein F, Dunphy PT, Fortney S, Riedesel ML. Simultaneous cerebrovascular and cardiovascular responses during presyncope. Stroke 1995;26:1794800 .

11 Imholz BPM, Wieling W, van Montfrans GA, Wesseling $\mathrm{KH}$. Fifteen years experience with finger arterial pressure monitoring: assessment of the technology. Cardiovasc Res 1998;38:605-16

12 Kosinsky D, Grubb BP, Temesy-Armos P. Pathophysiological aspects of neurocardiogenic syncope: current concepts and new perspectives. Pacing Clin Electrophysiol 1995;18: $716-24$.

13 Henderson MC, Prabhu SD. Syncope: current diagnosis and treatment. Curr Probl Cardiol 1997;22:244-51.

14 Vanderheyden M, Goethals M, Nellens P, Andries E, Brugada P. Different humoral responses during head-up tilt testing among patients with neurocardiogenic syncope. Am Heart $\mathcal{F}$ 1998;135:67-73.

15 Sorteberg W. Cerebral artery blood velocity and cerebral blood flow. In: Newell DW, Aaslid R, eds. Transcranial Doppler. New York: Raven Press, 1992:57-66. 
16 Tiecks FP, Lam AM, Aaslid R, Newell DW. Comparison of static and dynamic cerebral autoregulation measurements. Stroke 1995;26:1014-19.

17 Kawai K, Murthy G, Watenpaugh DE, Breit GA, Deroshia $\mathrm{CW}$, Hargens AR. Cerebral blood flow velocity in humans exposed to 24 hours of head-down tilt. $\mathcal{F}$ Appl Physiol 1993; 74:3046-51

18 Lindegaard KF, Lundar T, Wiberg J, Sjoberg D, Aaslid U, Nornes $\mathrm{H}$. Variations in middle cerebral artery blood flow investigated with noninvasive transcranial blood velocity measurements. Stroke 1987;18:1025-30.
19 Strandgaard S, Olesen J, Skinhoj E, Lasson NA. Autoregulation of brain circulation in severe arterial hypertension. lation of brain circul

20 Ladwig S, Ries S, Henning O, Valikovics A, Daffertshofer M, Pohlmann-Eden B. Combined electroencephalography and measurements of transcranial blood flow velocity during orthostatic testing - a new approach to assess syncope of unknown origin? Clin Auton Res 1997;7:305-9.

21 Grossi D, Nozzoli C, Roca ME, Santostasi R, Simone F. Head-up tilt for triggering and diagnosing syncope. Funct Neurol 1987;2:457-64.

\section{Making human cells make tumours}

If your put oncogenes into mouse cells you get tumours. If you do the same with human cells you get no tumours. Normal mouse cells, and most human tumour cells, express telomerase, and mouse cells have much longer telomeres than human cells. Human cells have been made to form tumours by using chemical or physical agents or an entire viral genome but now, for the first time, it has been done by inserting defined genes, including a telomerase gene, into the cells (William C Hahn and colleagues. Nature 1999;400:464-8).

Researchers in the USA used retroviruses as vectors to introduce three different genes into human embryonic kidney cells and fibroblasts; they were the hTERT gene, which encodes a subunit of telomerase, an oncogenic allele of $\mathrm{H}$-ras, and a simian virus large $\mathrm{T}$ oncoprotein. Cells that expressed all three genes showed tumour-like growth features and produced tumours when injected into nude mice. Cells expressing only two of the genes did not produce tumours.

The authors suggest that at least four different biochemical signalling pathways are involved (one more than in mice), one affected by ras, one by hTERT, and one by large T. Maintaining telomere length is probably necessary for human cells to develop into tumours. They do not speculate about the future implications of their research but presumably they are considerable.

ARCHIVIST 